\title{
Rethinking What Is Essential in the Office Visit Note
}

\author{
Stephen D. Persell, MD, MPH ${ }^{1,2}$ (1) and Heather L. Heiman, $M D^{3,4}$
}

'Division of General Internal Medicine and Geriatrics, Department of Medicine, Feinberg School of Medicine, Northwestern University, Chicago, IL, USA; ${ }^{2}$ Center for Primary Care Innovation, Institute for Public Health and Medicine, Feinberg School of Medicine, Northwestern University, Chicago, IL, USA; ${ }^{3}$ Division of Academic Internal Medicine and Geriatrics, Department of Medicine, University of Illinois College of Medicine, Chicago, IL, USA;

${ }^{4}$ Department of Medical Education, University of Illinois College of Medicine, Chicago, IL, USA.

J Gen Intern Med 36(11):3571-2

DOI: $10.1007 / \mathrm{s} 11606-021-06860-\mathrm{z}$

() Society of General Internal Medicine 2021

$\mathrm{T}$ he new ambulatory documentation rules from the Centers for Medicare and Medicaid Services (CMS), which took effect on January 1, are far from the most dramatic medical news of this new year. ${ }^{1}$ But these changes, which significantly update long-stagnant documentation requirements for officebased evaluation and management visits, deserve attention and applause. The rules attribute trust and value to the cognitive reasoning of the outpatient physician, and they may allow office visit notes to better serve the needs of physicians, patients, and colleagues.

Beginning this year, Current Procedural Terminology codes for evaluation and management services will be based either on the medical decision making needed for the patient or on total time spent in the care of the patient on that day. The history and exam need only be documented "as medically appropriate" and will no longer be used to determine billing level. $^{2}$

Sinsky and others have chronicled the inordinate time physicians spend on documentation - about $2 \mathrm{~h}$ of documentation and other desk work for every hour spent directly caring for patients. $^{3}$ This time burden may be unique to the USA; Holmgren et al. recently showed that electronic health record (EHR) users in the USA spent more time documenting than did their counterparts in other countries using the same EHR. ${ }^{4}$ Excessive documentation likely contributes to burnout and may displace activities more beneficial to patient care.

What really belongs in a note? Note readers may include patients, physician, and non-physician colleagues, and the author themself in the future. To meet the needs of these stakeholders, notes should be succinct, not full of clutter that introduces error and confusion. Notes should reflect the thought process of the author, their diagnostic and therapeutic plan, and the information and counseling the patient received.

Received January 27, 2021

Accepted April 26, 2021

Published online May 23, 2021
Notes should not duplicate content that is available elsewhere in the EHR, where it is more reliable (e.g., a radiology report which might be addended after the note is completed). The rule changes will advance all of these goals.

These changes have the potential to enrich the relationships between patients and their physicians. Freed from a billing incentive for providing a word-for-word transcription of the history and physical or for using a template that mechanically assembles a history of present illness from a set of radial buttons, physicians can focus on what they really need to know from the history. Data gathering remains crucial to patient assessment, but the note should be a roadmap of the visit, not a life-sized replica. ${ }^{5}$ Physicians may now be able to look up from their keyboard rather than frantically typing as patients reveal fears and secrets that need their physician's full attention. Physicians can stop asking non-sequitur questions to complete the full review of systems (which had ostensibly been evidence of a high level of complexity), and instead delve into a patient's social history or barriers to care, confirm understanding of risks and benefits of treatment, or solicit questions. The rule change formally acknowledges that important social needs (e.g., housing or food insecurity) may raise the complexity of an otherwise straightforward problem, a very welcome change.

Since the 21st Century Cures Act now ensures patients are able to read their clinicians' notes, ${ }^{6}$ physicians should be mindful of note attributes that may increase patient engagement in their medical care. Patients are ill-served by a mash-up of incomprehensible fill-in-the blank phrases or partially completed templates posing as their story. A shorter history and physical with just the essentials can be double-checked for accuracy, leaving time to write the assessment and plan. This section contains what the patient really cares to know: the name of the diagnosis or the medication that was recommended, the meaning of a study, or the reason a particular illness was thought unlikely.

The new rules also support the time physicians spend as stewards of the chart. Interoperability of EHRs across health systems has vastly increased the data available to the provider at the point of care, which has the potential to reduce waste and improve care. Record review before or after a visit can identify problems needing follow-up and studies already performed. Paying physicians for this formerly uncompensated review 
will increase the likelihood that they routinely perform this task.

A note is frequently the product of a single care provider, but patient care is now team-based. Burying critical information in a note hides it within a list of dozens or hundreds of such notes. Yet with the perception that billable documentation all needed to all be in the note, the note has become a dumping ground. Team-authored "wiki"-like elements such as the problem list, medication list, family history, and health maintenance sections are often unfinished or inaccurate, and they are often unnecessarily duplicated within the note. Remuneration for the time spent in chart upkeep should promote clearer and more accessible data about the patient in the shared spaces where all of the care providers expect to find it.

The new rules will require breaking some ingrained habits. While physicians might assume that more documentation will reduce risk in a malpractice case, the inclusion of greater amounts of data introduces potential for error. ${ }^{7}$ What is in the chart can be as damaging as what is missing. As notes become leaner, such notes will become a new standard of care.

The new CMS rules have some potential pitfalls. A busy provider may not be able to prepare for the clinic visit and finish documentation in 1 day, which is required for timebased billing. Extending billable time to the day before and the day after the appointment would more realistically fit many physicians' schedules. There is some risk that devaluing the history and physical might weaken data gathering, raising the likelihood of diagnostic error, especially among trainees newly developing clinical acumen. Clinical supervisors and administrators will need to make clear that collecting and documenting pertinent history and physical remains an expectation. For instance, a clinical note must provide enough information about the core elements of the history of present illness, review of systems, relevant past history, and physical exam to distinguish between causes of chest pain. Fleeting sharp chest pain in a 40-year-old without hypertension, diabetes, or smoking is entirely different from dull, exertional chest and neck pain in a 60-year-old with known diabetes and hypertension. Such detail must be present to judge whether a proposed clinical plan is appropriate. Clinicians responsible for supervising trainees should give feedback when key details are missing from the written record. Clinical documentation specialists who have been focused on assuring that the record supports the chosen level of billing may want to consider providing coaching to clinicians who shift too far in the direction of reduced documentation of the history and physical.

Overall, the opportunities well exceed the potential downsides. The new year offers an opportunity to re-imagine the EHR-based office note. Rather than a dumping ground, the note can be a canvas - for communication with patients and colleagues and for the clinical reasoning that should be valued as a core skill of the office-based clinician.

Corresponding Author: Stephen D. Persell, MD, MPH; Division of General Internal Medicine and Geriatrics, Department of Medicine, Feinberg School of Medicine, Northwestern University, Chicago, IL, USA (e-mail: spersell@nm.org).

\section{Declarations:}

Conflict of Interest: Dr. Persell receives unrelated research support from Omron Healthcare Co, LTD. and has previously received unrelated research support from Pfizer, Inc. He is a consultant to the RAND Corporation. Dr. Heiman reports receiving honoraria from the American Board of Internal Medicine. The authors received no funding for this work.

\section{REFERENCES}

1. Centers for Medicare and Medicaid Services. 42 CFR Parts 403, 409, 410, $411,414,415,416,418,424,425,489$, and 498 (November 15, 2019)

2. American Medical Association. CPT® Evaluation and Management. Available at https://www.ama-assn.org/practice-management/cpt/cpt-evaluation-and-management. Accessed December 31, 2020.

3. Sinsky C, Colligan $\mathbf{L}, \mathbf{L i} \mathbf{L}$, et al. Allocation of Physician Time in Ambulatory Practice: a Time and Motion Study in 4 specialties. Ann Intern Med. 2016;165(11):753-760. doi: https://doi.org/10.7326/M16-0961.

4. Holmgren AJ, Downing NL, Bates DW, et al. Assessment of Electronic Health Record Use Between US and non-US Health Systems. JAMA Intern Med. 2021;181(2):251-259. doi: https://doi.org/10.1001/jamainternmed. 2020.7071.

5. Martin SA, Sinsky CA. The map is not the territory: medical records and 21st century practice. Lancet. 2016;388(10055):2053-2056. doi: https:// doi.org/10.1016/S0140-6736(16)00338-X.

6. Office of the National Coordinator for Health Information Technology (ONC), Department of Health and Human Services (HHS). 21st Century Cures Act: Interoperability, Information Blocking, and the ONC Health IT Certification Program. 85 FR 25642. Published May 1, 2020. https://www. federalregister.gov/documents/2020/05/01/2020-07419/21st-centurycures-act-interoperability-information-blocking-and-the-onc-health-it-certification. Accessed April 10,2021.

7. Mangalmurti SS, Murtagh L, Mello MM. Medical Malpractice Liability in the Age of Electronic Health Records. N Engl J Med. 2010;363(21):2060-7. doi: https://doi.org/10.1056/NEJMhle1005210.

Publisher's Note: Springer Nature remains neutral with regard to jurisdictional claims in published maps and institutional affiliations. 